\title{
Reminiscence Therapy using Image-Based Rendering in VR
}

\author{
Emmanuelle Chapoulie Rachid Guerchouche $^{\S} \quad$ Pierre-David Petit ${ }^{\ddagger} \quad$ Gaurav Chaurasia ${ }^{\S}$

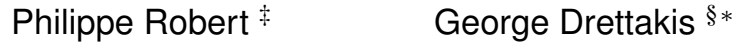 \\ $\S$ Inria, * CMRR Nice
}

\begin{abstract}
We present a novel VR solution for Reminiscence Therapy (RT), developed jointly by a group of memory clinicians and computer scientists. RT involves the discussion of past activities, events or experiences with others, often with the aid of tangible props which are familiar items from the past; it is a popular intervention in dementia care. We introduce an immersive VR system designed for RT, which allows easy presentation of familiar environments. In particular, our system supports highly-realistic Image-Based Rendering in an immersive setting. To evaluate the effectiveness and utility of our system for RT, we perform a study with healthy elderly participants to test if our VR system can help with the generation of autobiographical memories. We adapt a verbal Autobiographical Fluency protocol to our VR context, in which elderly participants are asked to generate memories based on images they are shown. We compare the use of our image-based system for an unknown and a familiar environment. The results of our study show that the number of memories generated for a familiar environment is higher than that for an unknown environment using our system. This indicates that IBR can convey familiarity of a given scene, which is an essential requirement for the use of VR in RT. Our results also show that our system is as effective as traditional RT protocols, while acceptability and motivation scores demonstrate that our system is well tolerated by elderly participants.
\end{abstract}

Keywords: Reminiscence Therapy, Image-Based Rendering, Virtual Reality, Immersive Environments, Autobiographical Fluency.

Index Terms: I.3.7 [COMPUTER GRAPHICS]: ThreeDimensional Graphics and Realism-Virtual reality / Applications

\section{INTRODUCTION}

Autobiographical memory (AM) is an essential component of human memory. In [9] it is defined as the mental representation of events from one's past (i.e., episodic AM) together with semantic information about the self. AM is one of the underlying processes of reminiscence therapy (RT). RT involves the discussion of past activities, events and experiences with another person or group of people, usually with the aid of tangible props such as photographs, household and other familiar items from the past. There is evidence of some improvement of functional ability in people with dementia using RT [35]. RT is one of the most popular psychosocial interventions in dementia care, and is highly rated by staff and participants. It is thus important to develop ways to improve RT using new technologies. In this work, we will focus on immersive Virtual Reality $(V R)$, which we define here as a setup with a large-screen, stereo 3D display, including head (and optionally hand/finger) tracking.

*E-mail: George.Drettakis@inria.fr; Inria authors are with REVES/Inria Sophia-Antipolis, http://www-sop.inria.fr/reves.

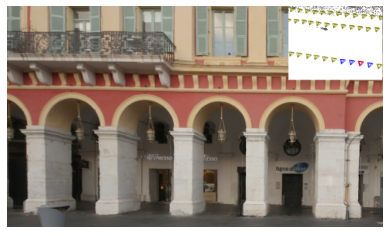

(a) Initial input camera

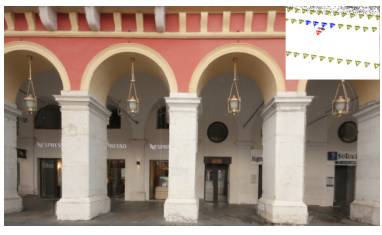

(c) Second novel view

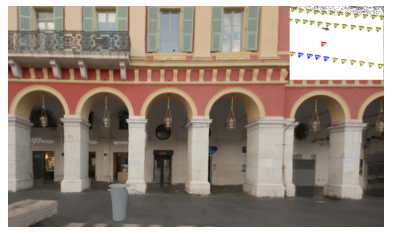

(b) First novel view

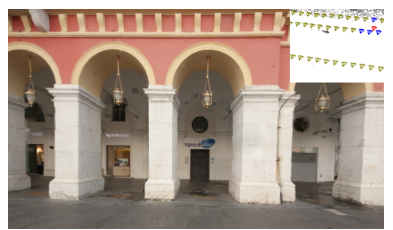

(d) Third novel view
Figure 1: (a) Image-based rendering with novel camera coincident with the input camera (red in inset); the result is identical to the input image. The user can move around freely in the area covered by the cameras. In (b)-(d) the method of [6] generates plausible novel views (red in inset) using the 4 views in blue.

There has been promising previous work in VR for memory treatments (e.g., $[3,19])$ In contrast to previous work however, VR support for RT in particular requires the presentation of environments which are familiar to the patient. Creating a realistic 3D model e.g., of a patient's neighborhood or landmarks of their city, is far too expensive using traditional manual modeling. In contrast, image-based modeling and rendering [33], provides a much easier way to achieve this goal, since only a small set of photographs is required as input. It is however only very recently [6] that imagebased rendering (IBR) has achieved a sufficient level of quality and efficiency to be suitable for free-viewpoint navigation, and thus for VR. The high level of realism of [6] can be seen in Fig. 1.

In this paper, we introduce an immersive VR system designed for RT, developed jointly by a group of memory clinicians and computer scientists. Based on the RT requirements listed above our system provides easy capture and display of familiar environments, by using IBR, and supports gesture-based navigation in the environment with finger tracking. We refer to the system as IVIRAGE Image-based VIrtual ReAlity with GEstures - from now on.

The potential clinical implications of IVIRAGE - if successful - are numerous. For example, the VEs provided by IVIRAGE are ecologically valid and generalizable, which is crucial for memory evaluation [21]. The realism and interactivity of IVIRAGE, also hold great promise for motivation and engagement [8], which are also key components of successul RT.

To evaluate the effectiveness and utility of IVIRAGE in RT, we performed a study with healthy elderly participants (mean age 66.84 years). The study investigates whether IVIRAGE can be useful for RT, by adapting a verbal Autobiographical Fluency test [13] to VR. In this test, elderly participants are asked to generate memories based on the images they see. We compare IVIRAGE for an unknown and a familiar environment to determine whether our approach can convey familiarity, and thus increase the number of gen- 
erated memories. We also investigate whether IVIRAGE is as effective in generating memories as current standard clinical practice.

Our contributions can thus be summarized as follows:

- We introduce the IVIRAGE system, demonstrating the first usage of free-viewpoint IBR in an immersive setting for RT.

- Our study shows that the number of autobiographical memories generated during the exposition to a familiar environment presented in IVIRAGE is higher than that for an unknown environment. This indicates that IBR can convey familiarity of a scene - an essential requirement for the use of VR in RT.

- Our study also shows that the IVIRAGE can be useful for RT, since acceptability scores indicate that the system is well tolerated by elderly patients, and by results that show that our system generates memories as effectively as traditional RT.

\section{Previous Work}

We first provide some additional background on autobiographical memory, and then briefly overview work on rehabilitation and VR, and in particular work concerning memory and the elderly. We also discuss related work on Image-Based Rendering (IBR).

Autobiographical Memory The retrieval of AM is a complex process, involving mental traces of past events and related semantic knowledge. These differ e.g., in content, imagery or emotional intensity. A fundamental dimension of an AM is its level of detail, which is thought to be a function of a person's database of memory traces, reconstructive processes, mental schemata, self goals and different retrieval strategies [10]. Episodic AM, especially the richness of details, is impaired early in the course of Alzheimer's disease or even in the preclinical phase, while semantic memories are spared until moderate stages, indicating a dissociation between both memory systems $[1,32]$. AM is used to develop a coherent sense of self, emotions and future plans [2]. It thus has a rich role in motivating behavior and in daily life. Many different methods have been used to study AM [20]; one approach is to stimulate memories using word, visual or other sensorial cues. We concentrate on verbal Autobiographical Fluency (AF) tests [13]. Such verbal fluency tests are widely used to assess language abilities and executive functioning [23]. These tasks involve rapid associative exploration and retrieval of words based on phonemic (e.g., starting with a specified letter of the alphabet) or semantic/categorical (e.g., animals) criteria over a brief timed interval.

VR and Neuropsychiatric Disturbances Therapy Early work in VR for cognitive assessment and rehabilitation is described in [27]; various theoretical and practical issues were discussed in [28]. The latter study underlines the importance of having graphics indistinguishable from the real world, demonstrating the utility of VR in this context. Treating phobias using VR has been investigated in different studies, e.g., [4]. VR was also used to treat Post Traumatic Stress Disorder [25]. VR has been used extensively for memory rehabilitation; the review in [3] demonstrates the positive effect of this technology. Recently, VR has been used to assess prospective memory using a driving simulator [19] with a large screen. Similarly, age effect on episodic memory was studied in a driving simulator [26]. Use of VR with the elderly imposes specific considerations. These have been studied early on (e.g., [22]), using VEs with stereo display and head/hand tracking. More recent results show that memory function can be improved with immersive audio-visual VR for the elderly [24]. Our approach follows this line of work, in proposing novel tools to improve RT for the elderly.

Image-Based Rendering View-dependent texture mapping [12] and the Unstructured Lumigraph [5] are early examples of IBR methods which allow interactive viewing. However, both suffer from disturbing visual artifacts if the reconstructed 3D geometry is not perfect. There have been many more recent methods which improve various aspects of IBR (see [33] for an overview).
However, true interactivity with quality at a level acceptable for VR has remained elusive, despite advances in the last few years (e.g., [15]). Most IBR systems can be classified into two types - proxy based $[5,15]$ and image warping approaches $[7,6]$. The former apply a view-dependent texture on the 3D model of the scene while the latter warp input images and blend the warped images to synthesize the final result. Proxy-based approaches require an accurate 3D model which is essentially impossible to obtain using state of the art 3D reconstruction [17]. The very recent work on depth synthesis and warp-based rendering [6], does provide rendering quality which is acceptable for some level of VR display. The quality of the rendered images - given specific restrictions which we develop below - is definitely acceptable for the type of immersive VR we will use in this study, despite some residual visual artifacts. The resulting realism is very high, as can be seen in Fig. 1 and the accompanying video, even in views away from the input photographs used to reconstruct the scene. We summarize this algorithm in Sec. 4.2. since it is central to our study.

\section{GoAls AND Hypotheses}

Our approach is to use IBR for episodic memory stimulation in an autobiographical fluency protocol. Navigating in a highly realistic Image-Based Virtual Environment (IBVE) opens up new possibilities for RT. In particular, our approach allows easy access to highly realistic representations of familiar and personalized environments, and provides a high level of interactivity.

We investigate whether our immersive system is useful in stimulating autobiographical memories in healthy elderly adults and whether the highly realistic environments provided by IVIRAGE are powerful enough to convey a sense of familiarity to participants. To achieve this goal we designed and ran an experiment in which we compare the number of AF responses obtained in a familiar IBVE to those obtained in an unknown IBVE. We hypothesize that the immersion in the familiar scene will result in a higher number of responses: an indication that IVIRAGE can convey familiarity.

We also investigate whether IVIRAGE is as effective as traditional RT, by presenting a static photograph of a familiar environment, since photographs of familiar sites are used extensively in RT. We test whether IVIRAGE can generate a similar number of memories as the standard approach. However, there is a significant distinction between the two: to allow in-place navigation, the photographs used for the IBVEs have a narrow field-of-view (FOV) to provide a scale 1 immersive experience. In contrast, photographs used in traditional AF usually have large FOVs (e.g., a panoramic view), with the potential to elicit many memories. This gives an advantage to the traditional RT condition compared to the IVIRAGE condition with the familiar environment; we thus expect the number of memories generated by the familiar IBVE to be less than or at best equal to the familiar photo.

To be successful, we need to satisfy two fundamental requirements. First, we need to provide a working system allowing navigation using IBR and second, we need to demonstrate that our system and protocols are well tolerated by elderly participants and can be as effective as traditional RT. In the following, we present our system and experimental results that satisfy these requirements.

\section{IVIRAGE: AN INTERACTIVE SyStem BASEd ON IBR FOR REMINISCENCE THERAPY}

The main goal for our interactive VR system for RT is to provide easy capture of familiar and personalized environments, while allowing realistic interactive display and navigation. The target hardware setup is a large screen with stereo display, and a high-end tracking system, which we describe next. We then describe the IBR approach of [6] we integrated and adapted to our system. 


\subsection{Hardware Setup}

The IBR algorithm [6] we use in our system is limited to a single screen (see supplemental material for more details); consequently, we only use the front wall of a BARCO iSpace for our experiments (see inset). The size of the screen is $320 \mathrm{~cm} \times 240 \mathrm{~cm}$ (resolution $1600 \times 1200$ ). The projectors use passive Infitec stereo via glasses, which are tracked using the ART tracking system (http: //www.ar-tracking.com). In addition, we use the ART wireless finger-tracking of the orientation of the hand and the position of the fingers. We use the three-finger (thumb-index-middle finger) version.

The fact that our participants are elderly (over 60 years old) implies specific precautions in the hardware setup. To avoid any risk of unsteadiness or falling, participants sat on a chair installed in front of the display screen, at a distance of $1 \mathrm{~m}$, during the experiments. A small stool is placed next to the chair for the experimenter.

To enrich the sensation of immersion in the VR environments, we added ambient spatialized 3D sound rendering over a 7.1 loudspeaker setup in the cube. We typically play sounds such as street, car and ambient noise, crowds walking and talking etc.

\subsection{Image-Based Capture, Rendering and Navigation}

To achieve the best possible visual quality, we adapted the approach of [6]. This approach is designed for free-viewpoint navigation in the scene, making it suitable for interactive VR.

The captured photographs are processed in order to synthesizes plausible depth, and thus compensate for lack of 3D geometry. The positions and orientations of the cameras which captured the images used for one of the scenes in this study are shown in Fig. 2.

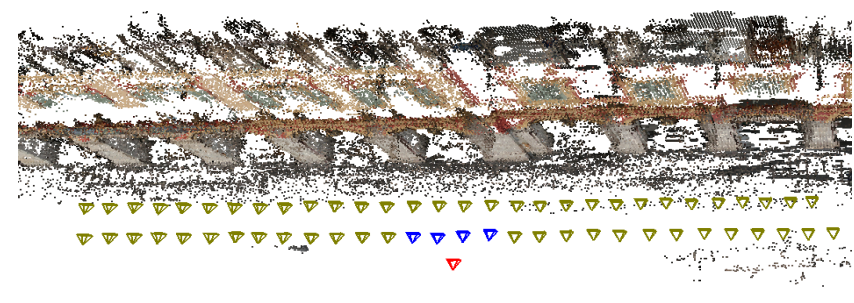

$\nabla \nabla \nabla \nabla \nabla \nabla \nabla \nabla \nabla \nabla \nabla \nabla \nabla \nabla \nabla \nabla \nabla \nabla \nabla \nabla \nabla \nabla \nabla \nabla \nabla \nabla \nabla \nabla \nabla \nabla$

Figure 2: Top view of camera positions for the capture of the FamIBVE (see Sec. 5.3). This set of cameras allows navigation in a significantly large portion of the scene $(75 \times 30 \mathrm{~m})$.

The approach in [6] was developed for desktop applications where the user navigates using a mouse. In our immersive space setup, the novel camera position is provided by the head tracker in real time. A description of the algorithmic changes required to adapt [6] to the immserive display are in the supplemental material.

While our IVIRAGE system provides very realistic images, like any image-based method it is restricted to representing content which actually exists in the input images. When the user leaves this region, visual artifacts appear. To avoid this, and to ensure that all participants navigate in the same regions, we limit navigation to the zone where artifacts are very small.

Our system supports gesture-based navigation in the immersive experimental conditions. The majority of participants had no previous VR or gaming expertise. For this reason, we added a training session in order to habituate the participants to the use of the fingertracking device. It consists of a session of grabbing and releasing virtual objects in the IBR environments; more details can be found in the supplemental material.

To navigate inside the IBVEs, participants have to point in the direction they want to follow with the index finger and perform a pinch gesture of the thumb and middle finger. Direction is specified by the palm orientation so that the pointing can be approximate, but we instruct participants to point for clarity and simplicity. The gesture is independent of hand location and thus participants can place their hand on their knees to avoid arm muscular fatigue. This is particularly important for our elderly participants. Visual feedback of their thumb, index and middle fingers is presented as red, green and blue cylinders respectively (see supplemental material).

\section{Experimental Methodology}

We next describe the assessment methodology procedure, which is an adaptation of traditional Autobiographical Fluency [13].

\subsection{Population}

The participants were 13 healthy elderly adults ( 5 Female and 8 Male, mean age $=66.84, \mathrm{SD}=4.33$ ) recruited by the clinical Memory Center research group. Each participant underwent a cognitive behavioral and motor assessment. Subjects not living in the city of experimentation, with a clinical history of neuropsychiatric disease or dementia, with a Mini Mental Score [16] lower than 28 (see Sec. 6.1) were excluded as well as subjects with dizziness or motor instability. All participants gave their informed consent before beginning the study. Ethical approval was received from the competent Ethics Committee.

\subsection{Autobiographical memory assessment}

In the AF test adapted for the experiment, participants had 2 minutes to generate as many memories as possible related to the environment they see, using short sentences. At the end of this time period the experimenter assessed the quality of the recollection using the Remember/Know procedure [18]. The subject was asked to indicate for each of his/her recollection if it is a Remember, i.e., conscious recollection of many vivid contextual details, such as "when" and "how" the information was learned or a Know, i.e., the sensation that the memory/event/environment has been seen before, but not being able to pin down the reason why.

The subjects verbal output was recorded during the experiment. The audio recordings were analyzed by a speech linguist independent from the design of our study. Derived scores include the Total number of memories and the number of Remember and Know recollections. In addition to the $\mathrm{AF}$, subjects also had to complete questionnaires assessing the acceptability and the quality of the experiences during the different conditions and a standard Presence questionnaire (see Sec. 6.3).

\subsection{Experimental Conditions}

We have four different experimental conditions. First, we have two baseline conditions: a control condition which is a grey-colored blank screen and a photograph of a familiar location, which corresponds to the use of photos for traditional AF. We then have the two conditions with IVIRAGE, namely an unknown and a familiar environment. The four conditions are shown in Fig. 3, (a)-(d).

Baseline Conditions. The first condition (a), which we refer to as Grey consists of blank, medium grey screens. The second condition (b), referred to as Familiar Photo (FamPhoto), is a wellknown location in the city of the experiment. Baseline conditions are displayed as simple images, without stereovision nor tracking.

IVIRAGE Conditions. Both IVIRAGE conditions are constructed and displayed with the method of [6]. We refer to the third condition (c) as Familiar IBVE (FamIBVE). It consists of an imagebased representation of a known landmark square in the city of the 


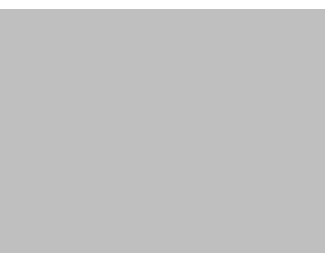

(a) Grey

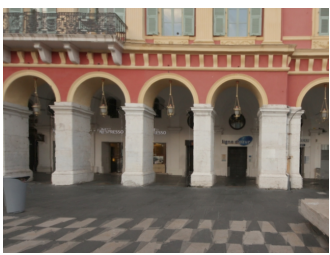

(c) FamIBVE

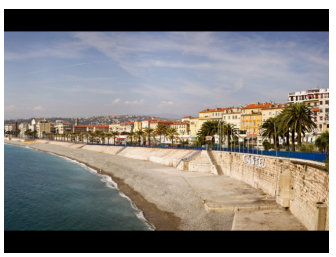

(b) FamPhoto

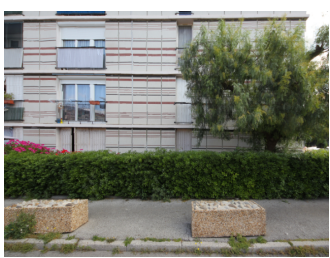

(d) UnknoIBVE
Figure 3: The four conditions used in our experiment.

experimentation. The fourth condition (d) is the Unknown IBVE (UnknoIBVE) which is captured in a public housing neighborhood, and contains buildings without recognizable features.

Capture of IBVEs. We took 90 and 35 photographs for the FamIBVE and UnknoIBVE scenes respectively. The placement of the cameras used for the first environment are shown in Fig. 2. The total time for photography, reconstruction and preprocessing is between 2-4 hours, while rendering is real-time.

\section{Experimental Procedure}

The experimental procedure has three main steps: inclusion, preparation and exposure to the different conditions.

\subsection{Clinical inclusion}

Clinical inclusion aims at verifying if the subject is a healthy adult and not a patient suffering from a memory disease. Before starting the experiments in the immersive space, each participant spends about 45 minutes with a clinician in a separate room.

After a motor clinical examination, the following tests are performed: Mini Mental Score [16], Short Cognitive Battery [30], Frontal Assessment Battery [14], and the Apathy Inventory [29].

This ensures that the participant is physically and mentally fit to participate in the experiment. If successful, the experimenter accompanies the participant to the immersive space. All participants succeeded the inclusion and took part in the study.

Before starting the experiment, we verify if participants are not stereo-blind by displaying a random-dot stereogram, and we perform a calibration procedure for finger tracking to adapt to each individuals' hand and finger size. The latter takes 2 minutes.

\subsection{Exposure to the environments}

The four environments are presented one after the other to each participant. "Grey" is always presented second since we do not expect the order of its presentation to influence performance. The other 3 environments are randomized in 6 different groups.

For the baseline conditions, the participant is presented with the photograph or blank screen for 15 seconds, and then the verbal fluency session begins.

For the IVIRAGE conditions, the session starts with the fingertracking system training session (see supplemental material for more details). The participant is then invited to navigate using the pointing gesture in the environment for about 45 seconds. At the end of the navigation, the verbal fluency test is performed.

We encourage the reader to view the accompanying video which illustrates the compelling experience provided by IVIRAGE.

\subsection{Post Exposure}

After the autobiographical fluency test each participant completed a set of questionnaires. (i) General questionnaire. A questionnaire was presented on a tablet to each participant at the end of each condition. The participant was asked whether the environment (except Grey) was recognized (YES/NO), and a continuous scale from 0 to 10 was used to rate the Familiarity with the environment (except Grey) and Emotion, Anxiety, Motivation, Security and Fatigue for all conditions. (ii) CyberSickness and Presence. For the IVI$R A G E$ conditions (UnknoIBVE and FamIBVE), we tested cybersickness with the 22-item questionnaire of [34], and Presence using the questionnaire (http://www.igroup.org/pq/ipq/ index. php) presented in [31].

\section{Results}

We present the results of our quantitative measurements as well as qualitative evaluation elements.

\subsection{Quantitative Evaluation}

Autobiographical Fluency Responses. The speech linguist recorded the number of responses and determined those that are conscious responses (Remember) or more vague recollections (Know) (Sec. 5.2). Only Remember values are relevant for our study. The median values for Grey, UnknoIBVE, FamIBVE and FamPhoto are respectively: 1, 3, 5 and 5.

Since the number of Remember responses is the most significant measure in the present study, Fig. 4 illustrates the number of such answers for each participant for the IVIRAGE conditions.

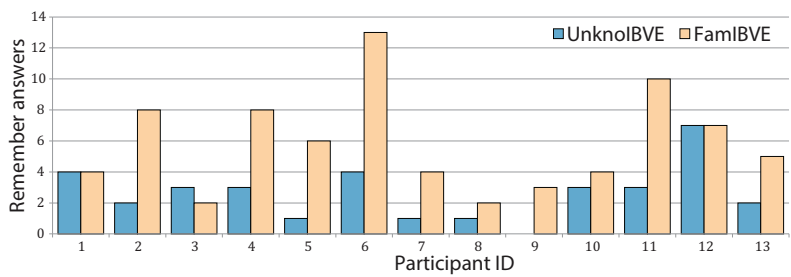

Figure 4: UnknoIBVE vs. FamIBVE: Remember answers.

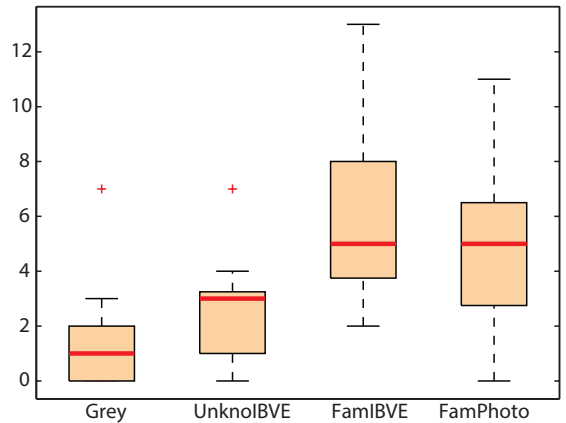

Figure 5: Remember data for the 4 conditions. The red bar is the median; we also show quartiles and distribution.

The Remember responses for the 4 conditions are shown in Fig. 5. We performed the non-parametric Friedman test [11] on this data. A significant main effect of condition was found for $R e$ member $\left(\chi^{2}=18.22, p=0.0004\right)$. No effect of the group order was found $\left(\chi^{2}=6.23, p=0.82\right)$.

We then ran a Wilcoxon Signed Ranks Test [11] to determine whether the differences between conditions are significant. For Remember, Grey is significantly different from FamIBVE ( $p=$ $0.0002)$ and from FamPhoto $(p=0.00056)$; UnknoIBVE is significantly different from FamIBVE $(p=0.004)$ and FamPhoto $(p=$ 0.01 ). No other significant differences were found. 
General Questionnaire. All the participants indicated that they recognized the FamIBVE but not the UnknoIBVE (except one participant who knows both environments (see Sec. 7.2)). The results from the familiarity question show a high score for the FamIBVE (Mean=7.92, SD=1.86) and for the FamPhoto (Mean=8.74, $\mathrm{SD}=1.44$ ) and a low score for the UnknoIBVE (Mean=3.28, $\mathrm{SD}=3.02$ ). The results for the Emotion, Motivation and Security are shown in Tab. 1.

Table 1: Acceptability and qualities of the experiences (Emotion $(E)$, Motivation $(M)$, Security $(S)$ ) during different conditions. $M n$ and $S D$ respectively refer to mean and standard deviation.

\begin{tabular}{|c|c|c|c|c|c|c|c|c|c|c|c|c|}
\hline / & \multicolumn{4}{|c|}{ Grey } & \multicolumn{3}{c|}{ UnknoIBVE } & \multicolumn{3}{c|}{ FamIBVE } & \multicolumn{3}{c|}{ FamPhoto } \\
\hline / & $E$ & $M$ & $S$ & $E$ & $M$ & $S$ & $E$ & $M$ & $S$ & $E$ & $M$ & $S$ \\
\hline$M n$ & 4.29 & 4.84 & 7.86 & 3.82 & 6.44 & 7.46 & 7.23 & 8.59 & 9.4 & 7.30 & 8.64 & 9.43 \\
\hline$S D$ & 3.54 & 3.14 & 3.00 & 3.27 & 3.41 & 3.28 & 1.56 & 1.3 & 0.47 & 1.98 & 0.99 & 0.38 \\
\hline
\end{tabular}

Emotion is highest in FamPhoto (Mean=7.30, $\mathrm{SD}=1.98$ ) and FamIBVE (Mean=7.23, 1.56), while it was low for Grey (Mean=4.29, $\mathrm{SD}=3.54)$ and UnknoIBVE $(\mathrm{Mean}=3.82, \mathrm{SD}=3.27)$. Mean Motivation is highest in familiar conditions (FamPhoto / FamIBVE) (Mean=8.64/8.59, SD=0.99/1.3) while mean Security for FamIBVE (Mean=9.7, SD=0.47) is on a par with FamPhoto (Mean=9.43, $\mathrm{SD}=0.38$ ).

We first ran a Levene normality test [11] for the condition data for Emotion, Motivation and Security. We found that only Motivation follows a normal distribution. We thus ran a two-way ANOVA test [11] on the Motivation data and a Friedman test on the Emotion and Security data. The results are as follows: there is a significant main effect of condition for Emotion $\left(\chi^{2}=13.64, p=0.0034\right)$ and Motivation $(F=5.56, d f=(3,12), p=0.0030)$; there is no effect for Security $\left(\chi^{2}=4.00, p=0.26\right)$. There is no significant effect of group for all these variables.

The Wilcoxon Signed Ranks Test showed significant differences for Emotion between Grey and FamIBVE $(p=0.01)$; Grey and FamPhoto $(p=0.013)$; UnknoIBVE and FamIBVE $(p=0.003)$; UnknoIBVE and FamPhoto $(p=0.0012)$. Using the same statistical test, Motivation is significantly higher for FamIBVE compared to Grey ( $p=0.018)$, FamPhoto compared to Grey $(p=0.0073)$ and UnknoIBVE compared to FamPhoto $(p=0.034)$.

Presence Questionnaire. The results of the Presence questionnaires have high overall scores (Median=5; scale 0-6) for General Presence and Spatial Presence [31]. The Experienced Realism and Involvement have medium scores (Median=3; scale 0-6) for both UnknoIBVE and FamIBVE. The Friedman test showed no significant main effect of condition $\left(\chi^{2}=1.32, p=0.87\right)$. Finally, participants did not experience sickness, fatigue or anxiety during the entire experiment across all conditions. This is shown by the data from the CyberSickness questionnaire (Mean=0.46, SD=0.1 for UnknoIBVE and Mean=1.42, SD=0.3 for FamIBVE on a scale of 0 to 88 ) as well as the Anxiety (Mean=2.3, $\mathrm{SD}=0.15$ on a scale of 0 to 10) and Fatigue (Mean $=0.87, \mathrm{SD}=0.27$ on a scale of 0 to 10 ) measures from the general questionnaire.

\subsection{Informal Qualitative Evaluation}

We did not perform a formal qualitative evaluation, but we report informal feedback and anecdotes from the experimental session.

All participants found the experience provided by IVIRAGE to be very interesting and stimulating; they expressed their satisfaction with many positive comments at the end of the sessions, both for interaction and navigation. One participant (number 3 in Fig. 4) had worked in the construction industry, and had actually worked on the construction of our unknown environment. Evidently, the un- known environment was actually familiar for this participant, which is reflected in the results of his fluency test.

In our familiar environment, there used to be a newsagent store which was recently closed, replaced by a fashion store with a much younger clientele. This newsagent had significant emotional value for the elderly population, and the vast majority of our participants commented on this issue, stating that they found this to be "a disgrace", "really a pity" etc.

Several participants commented that, despite the high level of realism, they found that the scene was "deserted". We provided the (true) explanation that the scenes are situated in the very early morning, and thus no people are present.

\section{Discussion}

From a clinical perspective the results of our study underline two main points which hold promise for the future: 1) It is possible to use IVIRAGE with elderly subjects and 2) that the system can stimulate conscious recollections of autobiographical memory.

Concerning the first point, the physical setup used (a chair or a bench) shows that this approach adapts well to populations e.g., with limited mobility. The responses to the questionnaires on Emotion, Motivation and Security, show that the technological setup was well tolerated by our participants. The fact that IVIRAGE can adapt the VE to the personal history and surroundings of participants holds particular promise from a clinical perspective, since it will be able to cognitively stimulate low mobility subjects.

Concerning the second point, previous work has already demonstrated the utility of VR for cognitive/memory processes (e.g., [3]). The increased number of memories with IVIRAGE for familiar vs. unknown scenes, shows that our system can help generate conscious autobiographical memories. This has several clinical implications. The goal of cognitive impairment treatment is to provide information on the subjects long term functional status.

In this field, the ecological validity or generalizability of neuropsychological test results, beyond the clinical environment, is a crucial component of the evaluative process [21]. The highly realistic environments of IVIRAGE are both ecological and generalizable, and give the additional opportunity to create an environment familiar to the patient and therefore closer to his/her real life.

Our study also suggests that it is possible to use VR in a therapeutic context. Our results indicate a positive effect of IVIRAGE on autobiographical memory which is a core component of RT. Our immediate future objective is to use our IVIRAGE system with patients in a clinical setting.

One of the great challenges in RT is whether different technologies maintain users motivation and engagement when confronting them with a repetitive series of training challenges, either cognitive or physical. This is of particular interest given that apathy i.e., the disorder of motivation, is the most frequent behavioral disturbance in elderly subjects with Alzheimer disease and related disorders. The interactivity and realism of IVIRAGE can improve motivation for training processes, with many positive consequences for patients. Engagement, defined as the act of being occupied or involved with an external stimulus [8] is the most challenging task for professional carers. Understanding individual interests may guide intervention parameters to improve engagement and potential benefits: the tailored environments we provide should thus be beneficial.

From a technical standpoint, the higher number of generated memories for FamIBVE compared to UnknoIBVE, is a very strong indication that IBR does convey familiarity in our immersive setting. The strong Presence scores also underline the ability of IBR to convey a sense of "being there". Despite the advantage (see Sec. 3) of the wide panoramic view of FamPhoto compared to the narrow FOV of FamIBVE, the number of responses is the same for both conditions, confirming the effectiveness of IVIRAGE for RT. We expect that the performance of FamIBVE will increase when the so- 
lution for multiple screens is available, since the FOV for the IBVE will be much larger.

Algorithmically, a new IBR algorithm for multiple screens is required, thus providing full immersion. While challenging, we are working on solutions to this. The addition of virtual humans to populate the scenes is another interesting challenge that should render the approach even more engaging and useful.

\section{Conclusions}

The results of our study shows that image-based techniques offer great promise for RT, and for VR in general. Our IVIRAGE system has numerous advantages over traditional 3D assets used in VR. The fact that only a few casual photographs are required to create a scene that can be used for VR is an advantage with very significant consequences. The level of realism obtained by the imagery, despite some residual artifacts, is at least as good that produced at great cost with traditional means e.g., manual modeling.

In conclusion, our study has given strong first evidence of the utility of IBR and close-to-natural gesture interaction for immersive VR systems, in the context of memory treatments and in particular RT. We are confident that our work will open the way to a much wider adoption of such technologies. The ease of creation of the IBVEs has the potential to not only greatly impact the use of VR for therapy, but also in other applications, since personalized assets can now be created and realistically displayed at low cost.

\section{ACKNOWLEDGEMENTS}

We acknowledge funding by the EU IP project VERVE (www.verveconsortium.eu) and the Region of PACA. We thank Autodesk for research and software donations, V. Lafont and A. Bouysset for help in the experiments, M. Marchal, A. Lécuyer and I. Viaud-Delmon for advice and J-S. Roo for photos.

\section{REFERENCES}

[1] F. Berna, P. Schönknecht, U. Seidl, P. Toro, and J. Schröder. Episodic autobiographical memory in normal aging and mild cognitive impairment: A population-based study. Psychiatry research, 200(2):807812, 2012.

[2] S. Bluck. Autobiographical memory: Exploring its functions in everyday life. Memory, 11(2):113-123, 2003.

[3] B. Brooks and F. Rose. The use of virtual reality in memory rehabilitation: current findings and future directions. NeuroRehabilitation, 18(2):147-157, 2003.

[4] M. Bruce and H. Regenbrecht. A virtual reality claustrophobia therapy system-implementation and test. In Proc. IEEE VR, 2009.

[5] C. Buehler, M. Bosse, L. McMillan, S. Gortler, and M. Cohen. Unstructured lumigraph rendering. In SIGGRAPH, ACM Proc., pages 425-432, 2001.

[6] G. Chaurasia, S. Duchene, O. Sorkine-Hornung, and G. Drettakis. Depth synthesis and local warps for plausible image-based navigation. ACM Trans. on Graphics (TOG), 32(3):30:1-30:12, 2013.

[7] G. Chaurasia, O. Sorkine, and G. Drettakis. Silhouette-aware warping for image-based rendering. Computer Graphics Forum, EGSR Proc., 30(4):1223-1232, 2011.

[8] J. Cohen-Mansfield, M. Dakheel-Ali, and M. S. Marx. Engagement in persons with dementia: the concept and its measurement. The American journal of geriatric psychiatry, 17(4):299, 2009.

[9] M. A. Conway. Memory and the self. Journal of memory and language, 53(4):594-628, 2005.

[10] M. A. Conway and C. W. Pleydell-Pearce. The construction of autobiographical memories in the self-memory system. Psychological review, 107(2):261, 2000.

[11] D. Cunningham and C. Wallraven. Experimental design: From user studies to psychophysics. AK Peters, Ltd., 2011.

[12] P. E. Debevec, C. J. Taylor, and J. Malik. Modeling and rendering architecture from photographs: A hybrid geometry-and image-based approach. In SIGGRAPH, ACM Proc., pages 11-20, 1996.
[13] B. H. Dritschel, J. Williams, A. D. Baddeley, and I. Nimmo-Smith. Autobiographical fluency: A method for the study of personal memory. Memory \& Cognition, 20(2):133-140, 1992.

[14] B. Dubois, A. Slachevsky, I. Litvan, and B. Pillon. The FAB a frontal assessment battery at bedside. Neurology, 55(11):1621-1626, 2000.

[15] M. Eisemann, B. D. Decker, M. Magnor, et al. Floating textures. Computer Graphics Forum, Eurographics Proc., 27(2):409-418, 2008.

[16] M. F. Folstein, S. E. Folstein, and P. R. McHugh. "Mini-mental state": a practical method for grading the cognitive state of patients for the clinician. Journal of psychiatric research, 12(3):189-198, 1975.

[17] Y. Furukawa and J. Ponce. Accurate, dense, and robust multi-view stereopsis. IEEE Trans. PAMI, 32(8):1362-1376, 2009.

[18] J. M. Gardiner, C. Ramponi, and A. Richardson-Klavehn. Experiences of remembering, knowing, and guessing. Consciousness and Cognition, 7(1):1-26, 1998.

[19] J. Gonneaud, P. Piolino, G. Lecouvey, et al. Assessing prospective memory in young healthy adults using virtual reality. In Disability, Virtual Reality, and Associated Technologies, Proc. of the 9th Int. Conf., pages 211-218, 2012.

[20] J. W. Griffith, J. A. Sumner, F. Raes, et al. Current psychometric and methodological issues in the measurement of overgeneral autobiographical memory. Journal of Behavior Therapy and Experimental Psychiatry, 43:S21-S31, 2012.

[21] M. Y. Kibby, M. Schmitter-Edgecombe, and C. J. Long. Ecological validity of neuropsychological tests: focus on the California Verbal Learning test and the Wisconsin card sorting test. Archives of Clinical Neuropsychology, 13(6):523-534, 1998.

[22] J. S. McGee, C. Van der Zaag, J. G. Buckwalter, et al. Issues for the assessment of visuospatial skills in older adults using virtual environment technology. CyberPsychology \& Behavior, 3(3):469-482, 2000.

[23] E. Miller. Verbal fluency as a function of a measure of verbal intelligence and in relation to different types of cerebral pathology. British Journal of Clinical Psychology, 23(1):53-57, 1984.

[24] G. Optale, C. Urgesi, V. Busato, et al. Controlling memory impairment in elderly adults using virtual reality memory training: a randomized controlled pilot study. Neurorehabilitation and neural repair, 24(4):348-357, 2010

[25] J. Pair, B. Allen, M. Dautricourt, et al. A virtual reality exposure therapy application for iraq war post traumatic stress disorder. In Proc. IEEE VR'06, pages 67-72, 2006.

[26] G. Plancher, V. Gyselinck, S. Nicolas, and P. Piolino. Age effect on components of episodic memory and feature binding: A virtual reality study. Neuropsychology, 24(3):379, 2010.

[27] G. Riva. Virtual reality in neuro-psycho-physiology: Cognitive, clinical and methodological issues in assessment and rehabilitation. IOS press, 1997.

[28] A. A. Rizzo, J. G. Buckwalter, U. Neumann, et al. Basic issues in the application of virtual reality for the assessment and rehabilitation of cognitive impairments and functional disabilities. CyberPsychology \& Behavior, 1(1):59-78, 1998.

[29] P. H. Robert, S. Clairet, M. Benoit, et al. The apathy inventory: assessment of apathy and awareness in alzheimer's disease, parkinson's disease and mild cognitive impairment. International journal of geriatric psychiatry, 17(12):1099-1105, 2002.

[30] P. H. Robert, S. P. Schuck, B. Dubois, et al. Screening for alzheimers disease with the short cognitive evaluation battery. Dementia and geriatric cognitive disorders, 15(2):92-98, 2003.

[31] T. Schubert, F. Friedmann, and H. Regenbrecht. The experience of presence: Factor analytic insights. Presence: Teleoperators and virtual environments, 10(3):266-281, 2001.

[32] U. Seidl, U. Lueken, P. A. Thomann, J. Geider, and J. Schröder. Autobiographical memory deficits in alzheimer's disease. Journal of Alzheimer's Disease, 27(3):567-574, 2011.

[33] H.-Y. Shum, S.-C. Chan, and S. B. Kang. Image-Based Rendering. Springer, 2007.

[34] I. Viaud-Delmon, Y. P. Ivanenko, A. Berthoz, and R. Jouvent. Adaptation as a sensorial profile in trait anxiety: a study with virtual reality. Journal of anxiety disorders, 14(6):583-601, 2000.

[35] B. Woods, A. Spector, C. Jones, et al. Reminiscence therapy for dementia. Cochrane Database Systematic Reviews, 2, 2005. 